\title{
Effect of Nickel Addition on Sintering Behavior and Electrical Conductivity of $\mathrm{BaCe}_{0.35} \mathrm{Zr}_{0.5} \mathrm{Y}_{0.15} \mathrm{O}_{3-\delta}$
}

\author{
Hyegsoon An****, Dongwook Shin**,****, and Ho-Il Ji*, \\ *High-temperature Energy Materials Research Center, Korea Institute of Science and Technology, Seoul 02792, Korea \\ **Department of Fuel Cells and Hydrogen Technology, Hanyang University, Seoul 04763, Korea \\ ***Division of Materials Science and Engineering, Hanyang University, Seoul 04763, Korea
}

(Received November 12, 2018; Revised November 14, 2018; Accepted November 16, 2018)

\begin{abstract}
The effect of different Ni-containing additives on the sintering behavior and electric conductivity of the proton conducting electrolyte $\mathrm{BaCe}_{0.35} \mathrm{Zr}_{0.5} \mathrm{Y}_{0.15} \mathrm{O}_{3-\delta}$ (BCZY5) was investigated. Ni-doped, NiO-added, and $\mathrm{BaY}_{2} \mathrm{NiO}_{5}(\mathrm{BYN}$-added (all 4 mol\%) BCZY5 samples were prepared by the solid state synthesis method and sintered at $1400^{\circ} \mathrm{C}$ for $6 \mathrm{~h}$. Among the three samples, the onset of densification was observed at the lowest temperature for NiO-added BCZY5, which is attributed to the formation of an intermediate phase at a low melting temperature. The BYN-added sample, where no consumption of the constitutional elements of the electrolyte was expected during sintering, exhibited the highest electrical conductivity whereas the doped sample had the lowest conductivity. The electrical conductivities at $500^{\circ} \mathrm{C}$ under humid argon atmosphere were measured to be $2.0,4.8$, and $6.2 \mathrm{mS} \cdot \mathrm{cm}^{-1}$ for $\mathrm{Ni}$-doped and NiO- and BYN-added samples, respectively.
\end{abstract}

Key words : Proton conducting electrolyte, Sintering aid, BCZY, Conductivity, PCFC

\section{Introduction}

A mong the different proton conducting electrolytes used in solid oxide fuel cells (SOFCs), Y-doped $\mathrm{BaCeO}_{3-}$ $\mathrm{BaZrO}_{3}(\mathrm{BCZY})$ has received increasing attention because of its high proton conductivity and chemical stability under humid as well as $\mathrm{CO}_{2}$ atmospheres. Protonic ceramic fuel cells (PCFCs), a new generation of SOFCs using proton conducting electrolytes, are expected to exhibit high performance and stability in the intermediate temperature range, which in turn, could improve the reliability and commercial viability of SOFCs. Hence, extensive research is being conducted to use BCZY as an electrolyte in a ceramic fuel cell. However, the refractory nature of BCZY is the principal hinderance to its practical application;, ${ }^{1,2)}$ for example, soak times of $10 \mathrm{~h}$ or longer at extremely high temperatures in the range $1600-1700^{\circ} \mathrm{C}$ are required for the densification of $\mathrm{BCZY}{ }^{3)}$ Moreover, this high temperature treatment is detrimental to the electrical conductivity of the material because of the evaporation or segregation of constituent elements such as $\mathrm{Ba}$ or $\mathrm{Y}$ from the interior of the crystal lattice. ${ }^{4}$ Hence, different strategies to improve the sinterability of the electrolyte, including the use of nano-sized electrolyte particles or partially leaving unreacted phases, have been attempted ${ }^{5,6)}$ However, despite these modifications, the temperature required for the total densification of the electro-

\footnotetext{
${ }^{\dagger}$ Corresponding author : Ho-Il Ji

E-mail : hiji@kist.re.kr

Tel : +82-2-958-5521 Fax : +82-2-958-5529
}

lyte is still high and is, therefore, not suitable for cell fabrication under real conditions. For anode-supported SOFCs, because two layers, i.e., thin electrolyte layers on the anode support, are co-fired, higher sintering temperature induces the formation of a substantially over-sintered anode structure as well as introduces defects such as a nonflat anode | electrolyte bilayer and a delaminated interface.

To overcome these disadvantages, transition metal oxides such as $\mathrm{CuO}, \mathrm{ZnO}$ and $\mathrm{NiO}$ are usually used as sintering aids for BCZY electrolyte densification. In principle, two approaches can be considered: Doping a metal oxide into the electrolyte lattice $\left(a \mathrm{MO}+\mathrm{BaCe}_{1-x-y} \mathrm{Zr}_{x} \mathrm{Y}_{y-a} \mathrm{O}_{3-\delta}\right.$ or $\left.\mathrm{BaCe}_{1-x-y} \mathrm{Zr}_{x-a} \mathrm{Y}_{y} \mathrm{O}_{3-\delta}\right)$ and adding an additional amount of metal oxide into the stoichiometric electrolyte $(a \mathrm{MO}+$ $\left.\mathrm{BaCe}_{1-x-y} \mathrm{Zr}_{x} \mathrm{Y}_{y} \mathrm{O}_{3-\delta}\right)$. Wang et al. investigated the two aforementioned approaches and confirmed that in both cases, the densification of BCZYs using the $\mathrm{ZnO}$ additive was achieved at a relatively low temperature of $1300^{\circ} \mathrm{C} .{ }^{7}$ For the former approach, because the total stoichiometry of the B site in $\mathrm{BCZY}$ is fixed to be 1 , and in the worst scenario, $\mathrm{Ba}$ and $\mathrm{Zn}$ in the BCZY lattice are evaporated in stoichiometric amounts in the case when the added $\mathrm{Zn}$ is above the solubility limit, no secondary phase is observed regardless of the amount of doping. On the other hand, for the latter approach, with increasing amount of the added metal oxide, the evaporation of $\mathrm{Ba}$ from the $\mathrm{BCZY}$ lattice is likely to increase owing to the extraction of $\mathrm{Ba}$ by $\mathrm{Zn}$, resulting in a secondary phase such as $\mathrm{Y}_{2} \mathrm{O}_{3}$ or $\mathrm{BaY}_{2} \mathrm{ZnO}_{5}$. However, while the electrical conductivity of $\mathrm{Zn}$-doped BCZY decreased sharply with increasing amount of added $\mathrm{Zn}$, that of $\mathrm{ZnO}$ - 
added BCZY was not significantly influenced by the amount of $\mathrm{ZnO}$. Thus, the blocking effect due to the formation of a secondary phase at the grain boundary is not the dominant factor that determines the electrical conductivity of the system. These results imply that the electrolyte composition and the formation of secondary phases are correlated in a complicated way and depend on the manner of addition of the sintering aid, which in turn, significantly affects the electrical properties of the electrolyte. In addition, Liu et al. reported a similar result wherein the $\mathrm{Zn}\left(\mathrm{NO}_{3}\right)_{2}$-added $\mathrm{BCZY}$ electrolyte in excess of stoichiometry promoted densification by forming the low melting $\mathrm{BaO}-\mathrm{ZnO}$ phase; a secondary phase of $\mathrm{Y}_{2} \mathrm{O}_{3}$ was also observed. ${ }^{8)}$ Recently, a solid state reactive sintering (SSRS) method was introduced, in which $\mathrm{NiO}$ was added to a mixture of the starting oxide powders $\left(\mathrm{BaCO}_{3}, \mathrm{ZrO}_{2}\right.$, and $\left.\mathrm{Y}_{2} \mathrm{O}_{3}\right)$ to simultaneously synthesize and sinter BZY.9) The proposed mechanism for SSRS includes the formation of the intermediate phase $\mathrm{BaY}_{2} \mathrm{NiO}_{5}(\mathrm{BYN})$ due to the reaction of the BZY raw material mixture with $\mathrm{NiO}$, which is then decomposed to promote the formation and sintering of Ni-doped BZY. Furthermore, because the doped $\mathrm{Ni}$ in the BZY lattice is ex-soluted from BZY in a reducing atmosphere, Ni-doped BZY returns to the BZY electrolyte. ${ }^{10)}$ Thus, it is expected that the formation of $\mathrm{BaY}_{2} \mathrm{NiO}_{5}$, which has been considered as an intermediate phase, serves as a sintering aid for the densification of BZY. Regarding the detailed mechanism that induces the enhanced sinterability, note that the intermediate liquid phase and/or a Ba-rich BZY phase is hypothesized to cause the accelerated sintering. A fraction of the inserted $\mathrm{Ni}$ is located at the interstitial site in the BZY lattice and is not substituted on the $\mathrm{B}$ site, which induces the extraction of $\mathrm{Ba}$ from the lattice, resulting in an Ba-excess environment out of the lattice. ${ }^{11)}$

The literature review presented above suggests that the compositional variation of the electrolyte and the formation of secondary phases are considerably dependent on the type of the sintering aid and the initial composition of the electrolyte, i.e., if it is a stoichiometric electrolyte or the one in which a cation is doped into the B site as a sintering aid. However, an in-depth understanding of the sintering behavior of systems with metal oxide doped or added in excess of stoichiometry as well as the reaction mechanism of the sintering aid is still lacking. Therefore, in this study, we first selected the nature of the sintering aid, which in this case is a Ni-containing species. Moreover, we have studied the differences in sintering behavior between Ni-doped BCZY $\left(\mathrm{BaCe}_{0.35} \mathrm{Zr}_{0.46} \mathrm{Ni}_{0.04} \mathrm{Y}_{0.15} \mathrm{O}_{3-\delta}, \mathrm{BCZY} 5 \mathrm{Ni}\right)$ and $\mathrm{NiO}$-added BCZY (4 mol\% $\mathrm{NiO}+\mathrm{BaCe}_{0.35} \mathrm{Zr}_{0.5} \mathrm{Y}_{0.15} \mathrm{O}_{3-\delta}$, NiO-BCZY). In addition, the formation of the BYN intermediate phase, which is indeed considered as the main species serving as the sintering aid during the actual cell fabrication step, was added to BCZY (4 mol\% $\mathrm{BaY}_{2} \mathrm{NiO}_{5}+\mathrm{BaCe}_{0.35} \mathrm{Zr}_{0.5} \mathrm{Y}_{0.15} \mathrm{O}_{3-\delta}$, BYN$\mathrm{BCZY5),} \mathrm{to} \mathrm{evaluate} \mathrm{if} \mathrm{there} \mathrm{was} \mathrm{a} \mathrm{deterioration} \mathrm{of} \mathrm{electrical}$ conductivity and whether its sintering behavior was different from the $\mathrm{Ni}$-doped and $\mathrm{NiO}$-added electrolytes. We believe that our results will guide the selection of the best approach to decrease the sintering temperature while avoiding a decrease in electrical conductivity of the electrolyte.

\section{Experimental Procedure}

$\mathrm{BaCe}_{0.35} \mathrm{Zr}_{0.5} \mathrm{Y}_{0.15} \mathrm{O}_{3-\delta}$ (BCZY5) was prepared using a conventional solid state reaction method following a procedure described in our previous article. ${ }^{12)}$ For $\mathrm{BaCe}_{0.35} \mathrm{Zr}_{0.46} \mathrm{Y}_{0.15} \mathrm{Ni}_{0.04} \mathrm{O}_{3-\delta}$ (BCZY5Ni), stoichiometric amounts of $\mathrm{BaCO}_{3}$ (Cerac, USA, 99.99\%), $\mathrm{CeO}_{2}$ (High purity chemical, Japan, 99.99\%), $\mathrm{ZrO}_{2}$ (Tosoh, Japan), $\mathrm{Y}_{2} \mathrm{O}_{3}$ (High purity chemical, Japan, 99.99\%) and $\mathrm{NiO}$ (Sumitomo, Japan) were weighed and ball-milled for $24 \mathrm{~h}$ in ethanol using zirconia balls as milling media. The mixture was calcined for 5 and $15 \mathrm{~h}$ at $1300^{\circ} \mathrm{C}$ and $1400^{\circ} \mathrm{C}$, and ball-milled for $48 \mathrm{~h}$. For $\mathrm{BaY}_{2} \mathrm{NiO}_{5}(\mathrm{BYN})$, the mixture of $\mathrm{BaCO}_{3}, \mathrm{NiO}$ and $\mathrm{Y}_{2} \mathrm{O}_{3}$ was calcined for $10 \mathrm{~h}$ at $1100^{\circ} \mathrm{C}$ and ball-milled for $48 \mathrm{~h}$. Crystal structures of the powders were analyzed using X-ray diffraction (XRD) in the $2 \theta$ range of $20^{\circ}-70^{\circ}$ with a scan rate of $1 \%$ min (D max $2500 /$ PC (Rigaku, Japan) and JADE 9.0 software).

Thermogravimetry (TG) and differential scanning calorimetry (DSC) were conducted simultaneously to assess the effect of Ni doping on the synthesis of BCZY5 powder. The powder mixtures of BCZY5 and BCZY5Ni prior to calcination, and $\mathrm{BaCO}_{3}$ powder were heated from room temperature up to $1450^{\circ} \mathrm{C}$ at the heating rate of $5^{\circ} \mathrm{C} / \mathrm{min}$ under flowing air with a flow rate of 200 sccm using a TGA set up (STA 409PC, NETZSCH, Germany).

To evaluate the effect of the different types of nickel addition on the sintering behavior of BCZY5, powder samples were prepared where equivalent mole percent of nickel or nickel containing species was added to the BCZY powder: 4 mol\% Ni-doped BCZY5 (BCZY5Ni), 4 mol\% NiO-added BCZY5 (NiO-BCZY5) and 4 mol\% BYN-added BCZY5 (BYN-BCZY5). All the powders were prepared by ball-milling and were then pelletized under a pressure of $100 \mathrm{MPa}$. The linear shrinkage of the samples was measured using a dilatometer (DIL 402C, NETZSCH, Germany) in the temperature range of $20-1500^{\circ} \mathrm{C}$ with a heating rate of $5^{\circ} \mathrm{C} / \mathrm{min}$ under $200 \mathrm{sccm}$ air flow.

A lab-made station was used to measure electrical conductivity. BCZY5Ni, NiO-BCZY5 and BYN-BCZY5 powders were pelletized using a cold isostatic press (CIP) under 200 $\mathrm{MPa}$ pressure and sintered at $1400^{\circ} \mathrm{C}$ for $6 \mathrm{~h}$. The obtained pellets were cut into rectangular bars $\left(0.5 \times 0.5 \times 1.4 \mathrm{~cm}^{3}\right)$ and all the sides of the bars were mirror-polished. A platinum wire was attached to the bar form a 4-probe configuration and platinum paste was brush-painted at the end of each side to promote electrical contact (the distance between the voltage probes was $0.8 \mathrm{~cm}$ ). For comparison, BCZY5 and BYN pellets were prepared by sintering at $1700^{\circ} \mathrm{C}$ for $10 \mathrm{~h}$ and $1350^{\circ} \mathrm{C}$ for $4 \mathrm{~h}$, respectively. The DC 4 probe measurement was conducted under dry and wet $\mathrm{Ar}$ $\left(p \mathrm{H}_{2} \mathrm{O}=2.3 \times 10^{3} \mathrm{~Pa}\right)$ using a current source (KE6220, 
Keithley, USA) and an electrometer (KE6154, Keithley, USA). The microstructure was investigated using a scanning electron microscope (SEM, Inspect F50, FEI).

\section{Results and Discussion}

With regard to Ni-doped BCZY5, we investigated whether $\mathrm{Ni}$ is indeed doped in BCZY5 lattice, and how it accelerates the formation of the BCZY5 phase. Fig. 1 shows TG/DSC profiles of the precursor powder mixtures for BCZY5 and BCZY5Ni prior to calcination; results for $\mathrm{BaCO}_{3}$ result are also plotted for comparison. For $\mathrm{BaCO}_{3}$, DSC analysis shows two peaks in the low temperature region which are identified to be due to the structural transformation of $\mathrm{BaCO}_{3}$ from orthorhombic to hexagonal at $820^{\circ} \mathrm{C}$, and then to the cubic phase at $970^{\circ} \mathrm{C}$. $\left.{ }^{9}\right)$ The peak around $1150^{\circ} \mathrm{C}$ corresponds to the decomposition of $\mathrm{BaCO}_{3}$ into $\mathrm{BaO}$ and $\mathrm{CO}_{2}$, which is in accordance with the rapid weight loss seen in TGA. In comparison, the DSC results for BCZY5 and BCZY5Ni precursors show that the peak corresponding to the decomposition of $\mathrm{BaCO}_{3}$ is no longer present whereas, new peaks appear at $\sim 1100^{\circ} \mathrm{C}$. This behavior is also observed during solid state reactive sintering (SSRS) of BZY with $\mathrm{NiO}$, and in this case, the signal is attributed to the formation of cubic perovskite phase. Therefore, we conclude that BCZY5 and BCZY5Ni are formed directly from the $\mathrm{BaCO}_{3}$ phase, and not from $\mathrm{BaO}$ that is formed by the decomposition of $\mathrm{BaCO}_{3}$. Surprisingly, although BCZY5 and BCZY5Ni show almost identical TG and DSC profiles except in the temperature range $900-1000^{\circ} \mathrm{C}$, the electrolyte phase in BCZY5Ni is formed at a lower temperature.

Figure 2 shows the diffraction patterns of BCZY5 and BCZY5Ni after calcination. When compared to BCZY5 without $\mathrm{Ni}$, a single phase of BCZY5Ni was relatively easy to be formed: monophasic BCZY5 is obtained only by a two-step process consisting of a first calcination step at $1300^{\circ} \mathrm{C}$ followed by a second calcination at $1400^{\circ} \mathrm{C}$. In contrast, a single phase of BCZY5Ni can be obtained in a single step

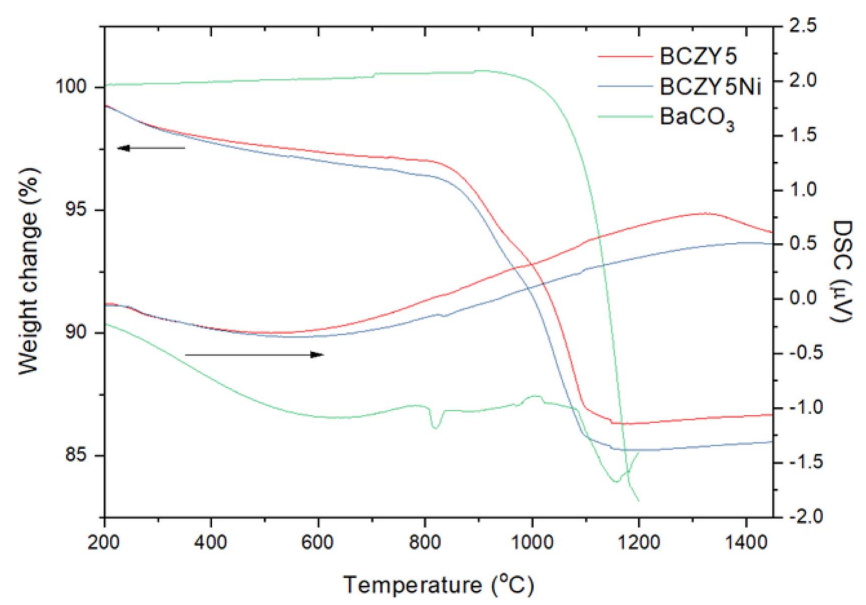

Fig. 1. TG/DSC profiles of BCZY5 precursor, BCZY5Ni precursor, and $\mathrm{BaCO}_{3}$.
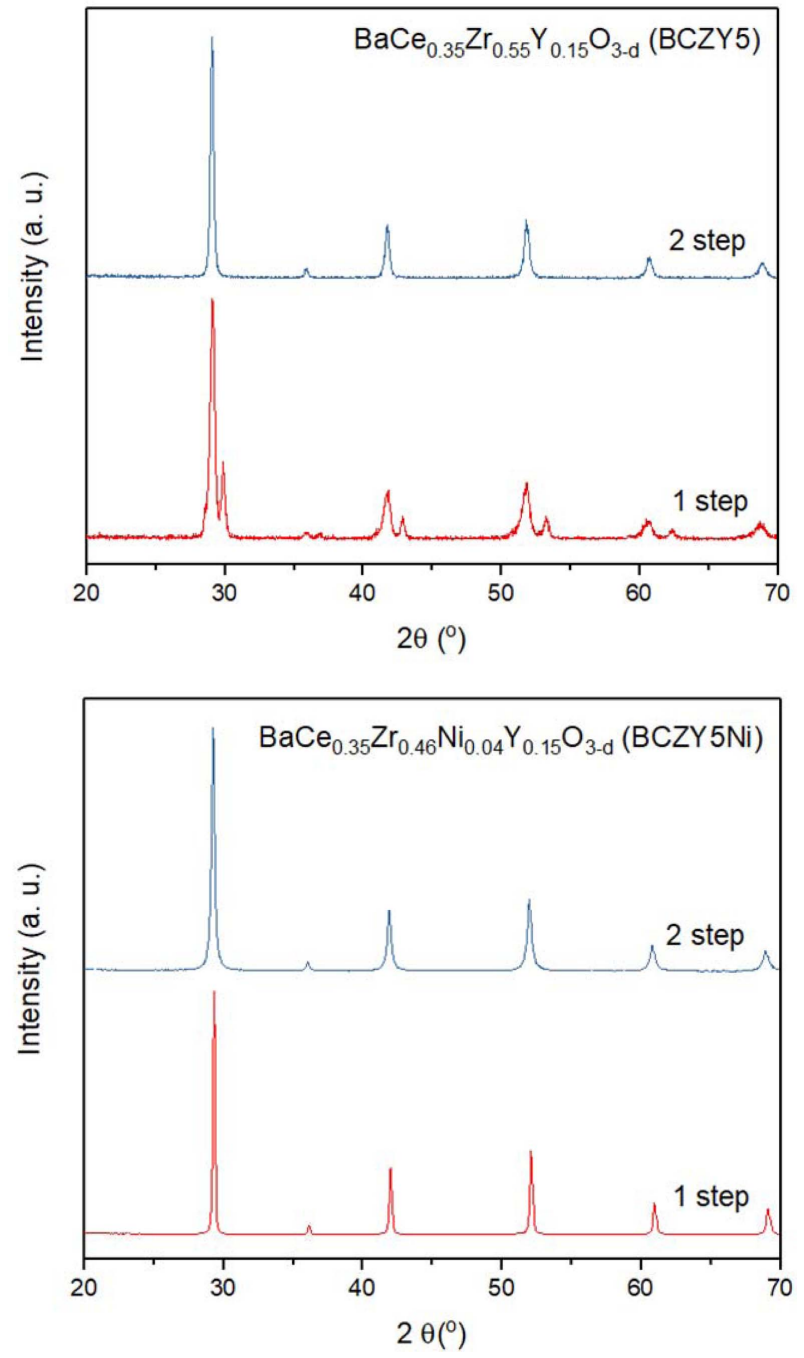

Fig. 2. XRD patterns of BCZY5 and BCZY5Ni powders calcined at $1300^{\circ} \mathrm{C}$ for $5 \mathrm{~h}$ (step 1 ) followed by a second calcination at $1400^{\circ} \mathrm{C}$ for $15 \mathrm{~h}$ (step 2).

calcination at $1300^{\circ} \mathrm{C}$ for $5 \mathrm{~h}$. Thus, there are two possible ways to promote the formation of a single phase. The first is by obtaining a new perovskite phase composed of BCZY5 and $\mathrm{Ni}$ as the dopant, i.e., BCZY5Ni, which is easily formed at a lower temperature than pure BCZY5. The second is through intermediates, e.g., $\mathrm{BaNiO}_{\mathrm{x}}(\mathrm{BN})$ and/or $\mathrm{BaY}_{2} \mathrm{NiO}_{5}$ (BYN), which are generated in the presence of $\mathrm{Ni}$ at a lower temperature than the calcination temperature of $1300^{\circ} \mathrm{C}$, which then accelerate the formation of BCZY5 phase and are subsequently incorporated into the BCZY5 lattice. Since any secondary or residual phases were not detected in the XRD patterns, it is inferred that Ni enters the BCZY5 lattice as a dopant. Rietveld refinement showed a change in lattice parameter due to $\mathrm{Ni}$ doping in BCZY5Ni (shown in Table 1).

Figure 3 represents the linear shrinkage curves of BCZY5 samples with $\mathrm{Ni}$ additives of various types, i.e., doping 4 mol\% Ni into the $\mathrm{Zr}$ site in BCZY5 (BCZY5Ni), addition of 4 mol\% NiO (NiO-BCZY5) and BYN (BYN-BCZY5) in stoi- 
Table 1. Rietveld Refinement Results for Sintered BCZY5, BCZY5Ni, NiO-BCZY5, and BYN-BCZY5 Powders

\begin{tabular}{|c|c|c|c|c|c|c|}
\hline & \multirow{2}{*}{ Crystal structure } & \multicolumn{3}{|c|}{ Lattice parameter } & \multirow{2}{*}{$\begin{array}{l}\text { Unit cell } \\
\text { volume }\left(\mathrm{A}^{3}\right)\end{array}$} & \multirow{2}{*}{$\begin{array}{c}\text { Pseudo-cubic } \\
\text { lattice parameter }(\mathrm{A})\end{array}$} \\
\hline & & $\mathrm{a}$ & $\mathrm{b}$ & $\mathrm{c}$ & & \\
\hline BCZY5 & \multirow{4}{*}{$\begin{array}{l}\text { Hexagonal } \\
\text { (R3-c) }\end{array}$} & 6.069 & & 14.889 & 474.956 & 4.294 \\
\hline BCZY5Ni & & 6.077 & & 14.928 & 477.431 & 4.301 \\
\hline NiO-BCZY5 & & 6.065 & & 14.911 & 475.069 & 4.294 \\
\hline BYN-BCZY5 & & 6.067 & & 14.907 & 475.132 & 4.294 \\
\hline
\end{tabular}

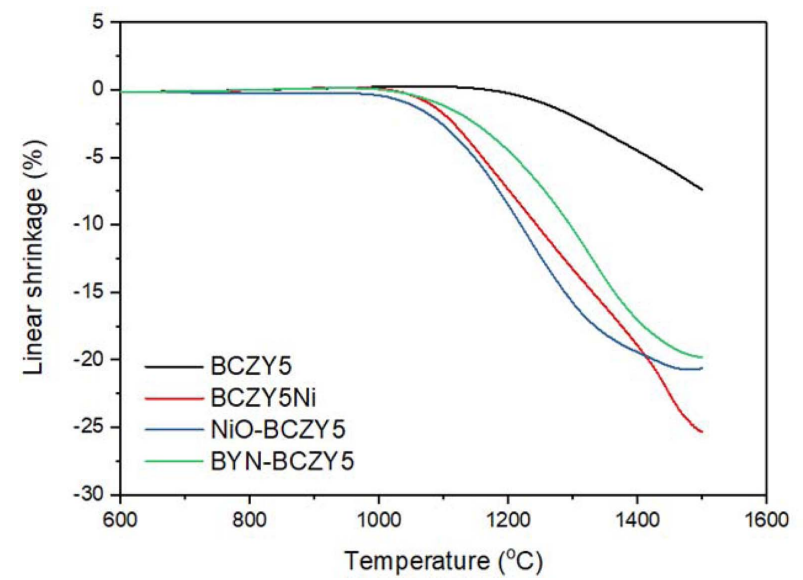

Fig. 3. Shrinkage curves of BCZY5 with 4 mol\% of Ni-doping, $\mathrm{NiO}$, and $\mathrm{BaY}_{2} \mathrm{NiO}_{5}$ sintering aids.

chiometric BCZY5. The shrinkage curve for BCZY5 is also plotted for comparison. As can be seen from the final shrinkage rate, $\mathrm{BCZY} 5$ without $\mathrm{Ni}$ shows $<10 \%$ shrinkage at $1500^{\circ} \mathrm{C}$, whereas the three samples with $\mathrm{Ni}$ show $>20 \%$ shrinkage. In particular, the shrinkage of NiO-BCZY5 started at the lowest temperature, while that of BYNBCZY5 lagged behind. The working mechanism of $\mathrm{Ni}$ added as a sintering aid has been reported to be according to the following reactions: ${ }^{13)}$

$$
\begin{aligned}
& \mathrm{Ba}_{1-\alpha} \mathrm{CZY}+\alpha \mathrm{BaO}+\mathrm{NiO} \rightarrow \mathrm{Ba}_{1-\alpha} \mathrm{CZY}+\alpha \mathrm{BaNiO}_{\mathrm{x}} \\
& \quad+(1-\alpha) \mathrm{NiO} \\
& \mathrm{Ba}_{1-\alpha} \mathrm{CZY}_{1-2 \alpha}+\alpha \mathrm{BaNiO}_{\mathrm{x}}+\alpha \mathrm{Y}_{2} \mathrm{O}_{3}+(1-\alpha) \mathrm{NiO} \rightarrow \\
& \mathrm{Ba}_{1-\alpha} \mathrm{CZY}_{1-2 \alpha}+\alpha \mathrm{BaY}_{2} \mathrm{NiO}_{5}+(1-\alpha) \mathrm{NiO}
\end{aligned}
$$

In Equations (1-2), $\mathrm{NiO}$ consumes $\mathrm{Y}$ and/or Ba in BCZY to form $\mathrm{BaNiO}_{\mathrm{x}}(\mathrm{BN})$ and $\mathrm{BaY}_{2} \mathrm{NiO}_{5}(\mathrm{BYN})$, and these phases promote densification by rearranging particle packing or enhancing element diffusion at the grain boundary. Thus, the reason for the difference in sintering behavior between $\mathrm{NiO}-\mathrm{BCZY} 5$ and BYN-BCZY5 is presumably related to whether or not the $\mathrm{BN}$ phase with a lower melting point $\left(1240^{\circ} \mathrm{C}\right)$ is formed prior to the formation of BYN. ${ }^{14)}$ As compared to $\mathrm{BN}, \mathrm{BYN}$ has a relatively high melting point
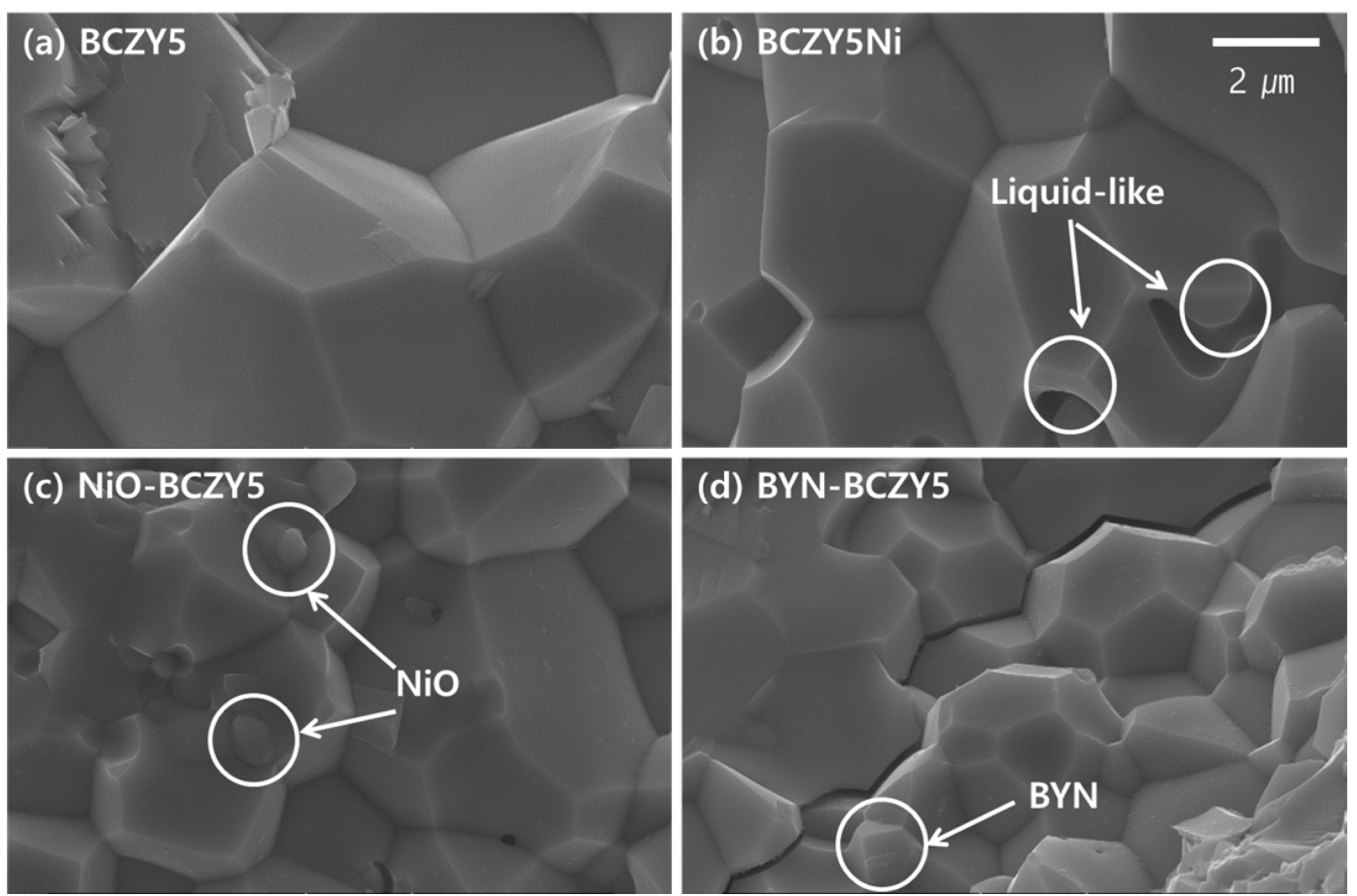

Fig. 4. SEM micrographs of fractured (b) Ni-doped, (c) NiO-added and (d) BYN-added BCZY5 pellets sintered at $1400^{\circ} \mathrm{C}$ for $6 \mathrm{~h}$. For comparison, (a) BCZY5 without Ni sintered at $1700^{\circ} \mathrm{C}$ for $10 \mathrm{~h}$ is presented. 
$\left(1450^{\circ} \mathrm{C}\right)$, as a result of which, densification starts at a higher temperature. However, BYN-added BCZY5 is expected that a deterioration in electrical conductivity is suppressed because of the absence of a reaction with the electrolyte.

In the case of BCZY5Ni, the observed shrinkage curve is in between the Ni-added results, below $1400^{\circ} \mathrm{C}$. Guo et al., explained the effect of sintering aid addition on the sintering behavior of BCZY. ${ }^{15)}$ As shown above for NiO-BCZY5 and BYN-BCZY5, when a sintering aid is added to the BCZY matrix, an intermediate phase having a low melting point is formed through reaction with $\mathrm{Y}$ and/or $\mathrm{Ba}$, which promotes densification. When the sintering additive is added to the BCZY lattice as a dopant, sintering is promoted due to the formation of a dopant-containing perovskite, which in turn, has a lower sintering temperature. The reaction of the dopant in the NiO-BCZY5 system can be represented by the following equation (3):

$$
\mathrm{BCZY}+\mathrm{NiO} \rightarrow \mathrm{BCZ}_{1-\alpha} \mathrm{Y}_{1-\beta} \mathrm{Ni}_{\alpha+\beta}+\alpha \mathrm{ZrO}_{2}+\beta \mathrm{Y}_{2} \mathrm{O}_{3}
$$

However, a recent report has indicated that the solubility of $\mathrm{NiO}$ in the perovskite is limited to $<1$ at.\% and the dopant is likely to be located at the interstitial sites rather than in the lattice. ${ }^{11)}$ Thus, the observation that the shrinkage curve of BCZY5Ni differs from that of NiO-BCZY5 can be explained to be due to the different ways by which the sintering aid promotes densification. This difference in sintering mechanism is evident not only in sintering behavior but also in the corresponding microstructures. Fig. 4 shows the cross-sectional SEM image of BCZY5 sintered at $1400^{\circ} \mathrm{C}$ for $6 \mathrm{~h}$ with addition of the sintering aid. For $\mathrm{NiO}$ (Fig. 4(c)) and BYN (Fig. 4(d)), dense microstructures were observed even at temperatures that were $300^{\circ} \mathrm{C}$ lower than the sintering temperature of BCZY5 without Ni. Moreover, from XRD analysis results, residues located at the grain boundary in SEM images could be assigned to the sintering aids, $\mathrm{NiO}$ and BYN (Fig. 5). The difference in sintering behavior caused by the melting point difference between $\mathrm{BaNiO}_{\mathrm{x}}$ and $\mathrm{BYN}$ also induces a difference in grain size. NiO-BCZY5 showed grain sizes in the $3 \sim 5 \mu \mathrm{m}$ range, which is equivalent to that of BCZY5 sintered $1700^{\circ} \mathrm{C}$, while the grain size of $\mathrm{BYN}-\mathrm{BCZY} 5$ remains at $2 \mu \mathrm{m}$. For proton-conducting materials, a large grain size is preferred, because it can minimize the grain boundary resistance. Therefore, if $\mathrm{BaNiO}_{\mathrm{x}}$ with a low melting point is applied as a sintering additive instead of BYN, large grains can be obtained, while at the same time avoiding consumption of the elements in the electrolyte.

BCZY5Ni showed a grain size similar to that of NiOBCZY5, but the presence of a liquid-like phase was observed in a few grains. There are two possible explanations for this phenomenon. One is that the intermediates such as $\mathrm{BaNiO}_{\mathrm{x}}$ are generated from $\mathrm{BCZY} 5 \mathrm{Ni}$, which accelerate sintering and subsequently evaporate or dissolve into the matrix during the sintering process. A similar phenomenon involving pore formation induced by the vaporization of barium and a transition metal has been reported in $\mathrm{Zn}$-doped

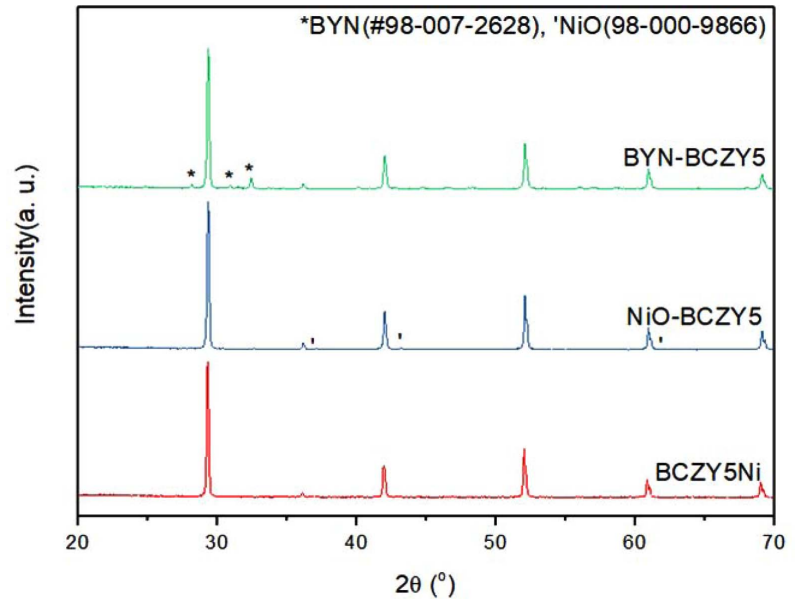

Fig. 5. XRD patterns of BCZY5 with $\mathrm{NiO}, \mathrm{BYN}$-added and BCZY5Ni. All the samples were sintered at $1400^{\circ} \mathrm{C}$ for $6 \mathrm{~h}$.

BCZY; this behavior intensified as the amount of the transition metal dopant was increased. ${ }^{7)}$ The other explanation is that the intermediate phase, which remains stable during the synthesis of BCZY5Ni, evaporates or dissolves into the matrix during sintering. XRD patterns of BCZY5Ni in Figure 5 show no intermediate phases. Moreover, results from Rietveld refinement (Table 1) show that the lattice constant of BCZY5Ni is increased from reference value unlike the other three samples The lattice constant is expected to decrease when smaller sized $\mathrm{Ni}(0.69 \AA)$ substitutes for the larger $\mathrm{Zr}(0.72 \AA)$, but it has been reported that the BCZY lattice is expanded when a small amount of transient metal is doped. ${ }^{16)}$ This could be due to the non-random distribution of the doped transition metal in the lattice. $\mathrm{BO}_{6}$ octahedra substituted by the transient metal are preferentially surrounded by $\mathrm{YO}_{6}$ octahedra to compensate for the higher electronegativity of the transition metal, which leads to an increase in the lattice constant. Therefore, it can be assumed that the liquid-like microstructure of BCZY5Ni shown in Fig. 5 is most probably due to the evaporation or dissolution during sintering, of the sintering accelerator generated from the $\mathrm{BCZY} 5 \mathrm{Ni}$ matrix.

Figure 6(a) shows the results of electrical conductivity measurements on sintered samples. The measurement was conducted in humidified argon atmosphere because under these conditions, a proton defect is generated by a hydration reaction in which, an oxygen vacancy is occupied by water vapor. BCZY5 without the sintering additive showed the highest electrical conductivity, followed by BYN-BCZY5, NiO-BCZY5 and BCZY5Ni. The activation energies of BCZY5, BYN-BCZY5, NiO-BCZY5 and BCZY5Ni were, respectively, $0.23 \mathrm{eV}, 0.25 \mathrm{eV}, 0.29 \mathrm{eV}$ and $0.39 \mathrm{eV}$ in the intermediate temperature range. It is interesting to note that BYN-BCZY5 showed a higher electrical conductivity than NiO-BCZY5, but this was only a slight increase, even though there was no deficiency of $\mathrm{Ba}$ or $\mathrm{Y}$ in the electrolyte, which has a detrimental effect on the conductivity. This is 


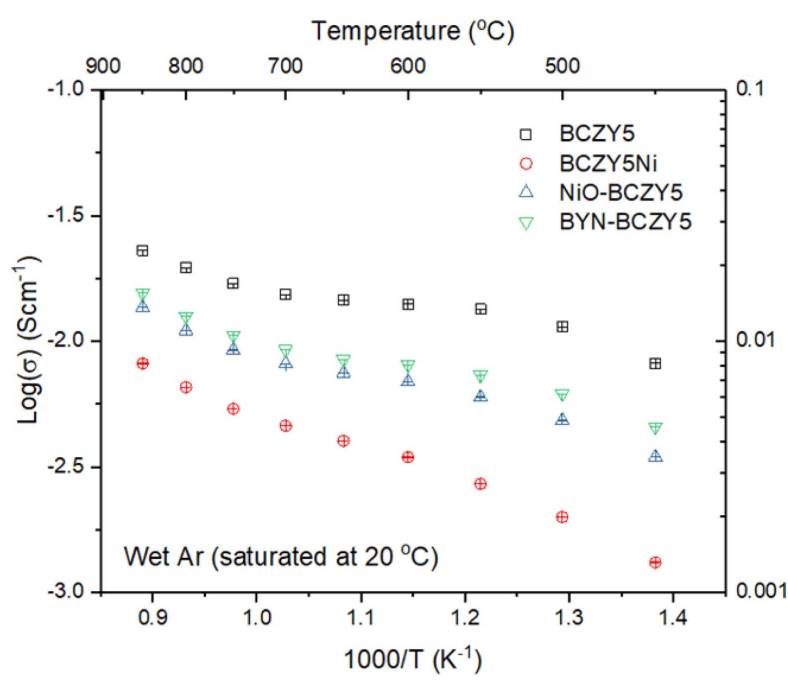

BCZY5 at $500^{\circ} \mathrm{C}$. This is expected to be solely due to the effect of Ni doping, since adequate grain growth of BCZY5Ni occurred (Fig. 4) and secondary phases were not observed in the XRD pattern (Fig. 5). Concerning the effect of Ni doping on the electrical conductivity of BCZY, it has been reported that addition of $1-2 \mathrm{~mol} \%$ of a sintering aid strongly reduces the grain and grain boundary electrical conductivity. ${ }^{16)}$

In summary, the sintering behavior, microstructure, and electrical properties of Ni-doped (BCZY5Ni), NiO-added (NiO-BCZY5), and BYN-added (BYN-BCZY5) electrolytes have been investigated. In the case of doping, it is most probable that an intermediate phase is generated from the BCZY5Ni matrix during sintering, which subsequently evaporates or dissolves into the matrix. In the case where $\mathrm{NiO}$ is added externally, elemental $\mathrm{Y}$ and/or $\mathrm{Ba}$ are extracted from the BCZY5 lattice to form the $\mathrm{BN}$ and/or BYN phases via reaction with a fraction of the added $\mathrm{NiO}$. The remaining $\mathrm{NiO}$ is localized at the grain boundary region after sintering. In the case of externally added BYN, it is expected that there is no deficiency of $\mathrm{Ba}$ or $\mathrm{Y}$ in the electrolyte, and the BYN phase remains at the grain boundary after sintering. The presence of residues and element deficiency of the electrolyte affect its electrical properties: Among these effects, Ni doping in the electrolyte results has the greatest deleterious effect. Elemental deficiency induced by $\mathrm{NiO}$ is next, followed by the effect of Ni-doping. BYN addition also results in a decrease in conductivity probably owing to the presence of BYN residue at the grain boundary and the higher grain boundary density. Nevertheless, among the range of methods currently available, external addition of $\mathrm{BYN}$ is the best approach to decrease the sintering temperature while minimizing the decrease in electrical conductivity.

\section{Conclusions}

and without sintering aid under dry and wet Ar. (b) Electrical conductivity of $\mathrm{BaY}_{2} \mathrm{NiO}_{5}$ under dry/wet air/Ar atmosphere.

probably due to the decrease in grain boundary resistance resulting from an increase of grain size of the NiO-BCZY5 sample, which compensates for the reduction in electrical conductivity induced by the consumption of the constituent elements in the electrolyte; as a result, the observed conductivity is similar to that of BYN-BCZY5. In addition, both methods leave residual $\mathrm{NiO}$ or $\mathrm{BYN}$ phases at the grain boundaries, which may serve as blocking layers for proton transport. Fig. 6(b) shows the electrical conductivity of BYN under humidified/dry air/argon atmosphere. Since the conductivities under humidified and dry atmospheres do not show a significant difference, it is inferred that BYN at the grain boundaries may serve as a blocking layer for proton transport. Furthermore, it has been reported that Sr-substituted BYN is an oxygen ion conductor. ${ }^{17)}$ Fig. 6(a) shows that the lowest electrical conductivity was observed for BCZY5Ni. For example, the electrical conductivity of BCZY5Ni is one order of magnitude lower than that of

The changes in electrical conductivity and sintering behavior of $\mathrm{BaCe}_{0.35} \mathrm{Zr}_{0.5} \mathrm{Y}_{0.15} \mathrm{O}_{3-\delta}$ (BCZY5) powders were investigated with respect to the formation of Ni-containing intermediate phases during sintering. Regardless of the mode of addition, the different additives promoted the sintering of BCZY5, and the densities obtained after sintering at $1400^{\circ} \mathrm{C}$ were all above $95 \%$. As compared to $\mathrm{NiO}$ addition (NiO-BCZY5) and Ni doping (BCZY5Ni), NiO addition was confirmed to be more beneficial to achieve the accelerated densification of BCZY5. The electrical conductivities of the un-doped BCZY5s (NiO-BCZY5 and BYN-BCZY5) were similar to that of pristine BCZY5. In addition, BYN-BCZY5 showed the highest electrical conductivity by avoiding the consumption of elements such as Ba and Y in BCZY5.

\section{Acknowledgments}

This work was supported by the Technology Development Program to Solve Climate Changes of the National Research Foundation of Korea funded by the Ministry of Science, ICT 
\& Future Planning (2017M1A2A2044982). H.A. thanks the Manpower Development Program for Energy supported by the Ministry of Knowledge and Economy.

\section{REFERENCES}

1. E. D. Wachsman and K. T. Lee, "Lowering the Temperature of Solid Oxide Fuel Cells," Science, 334 [6058] 935-39 (2011).

2. K. D. Kreuer, "Proton-Conducting Oxides," Annu. Rev. Mater. Res., 33 333-59 (2003).

3. K. Katahira, Y. Kohchi, T. Shimura, and H. Iwahara, "Protonic Conduction in Zr-substituted $\mathrm{BaCeO}_{3}$," Solid State Ionics, 138 [1-2] 91-8 (2000).

4. P. Babilo, T. Uda, and S. M. Haile, "Processing of YttriumDoped Barium Zirconate for High Proton Conductivity," J. Mater. Res., 22 [5] 1322-30 (2007).

5. Z. M. Zhong, "Stability and Conductivity Study of the $\mathrm{BaCe}_{0.9-\mathrm{x}} \mathrm{Zr}_{\mathrm{x}} \mathrm{Y}_{0.1} \mathrm{O}_{2.95}$ Systems," Solid State Ionics, 178 [3-4] 213-20 (2007).

6. Y. Yamazaki, R. Hernandez-Sanchez, and S. M. Haile, "High Total Proton Conductivity in Large-Grained YttriumDoped Barium Zirconate," Chem. Mater., 21 [13] 2755-62 (2009).

7. H. Wang, R. R. Peng, X. F. Wu, J. L. Hu, and C. R. Xia, "Sintering Behavior and Conductivity Study of YttriumDoped $\mathrm{BaCeO}_{3}-\mathrm{BaZrO}_{3}$ Solid Solutions Using $\mathrm{ZnO}$ Additives," J. Am. Ceram. Soc., 92 [11] 2623-29 (2009).

8. Y. Liu, Y. M. Guo, R. Ran, and Z. P. Shao, "A Novel Approach for Substantially Improving the Sinterability of $\mathrm{BaZr}_{0.4} \mathrm{Ce}_{0.4} \mathrm{Y}_{0.2} \mathrm{O}_{3-8}$ Electrolyte for Fuel Cells by Impregnating the Green Membrane with Zinc Nitrate as a Sintering Aid," J. Membr. Sci., 437 189-95 (2013).

9. J. H. Tong, D. Clark, L. Bernau, M. Sanders, and R. O'Hayre, "Solid-State Reactive Sintering Mechanism for Large-Grained Yttrium-Doped Barium Zirconate Proton Conducting Ceramics," J. Mater. Chem., 20 [30] 6333-41
(2010).

10. C. C. Duan, R. J. Kee, H. Y. Zhu, C. Karakaya, Y. C. Chen, S. Ricote, A. Jarry, E. J. Crumlin, D. Hook, R. Braun, N. P. Sullivan, and R. O'Hayre, "Highly Durable, Coking and Sulfur Tolerant, Fuel-Flexible Protonic Ceramic Fuel Cells," Nature, 557 217-22 (2018).

11. D. Han, K. Shinoda, S. Tsukimoto, H. Takeuchi, C. Hiraiwa, M. Majima, and T. Uda, "Origins of Structural and Electrochemical Influence on Y-doped $\mathrm{BaZrO}_{3}$ HeatTreated with $\mathrm{NiO}$ Additive," J. Mater. Chem. A, 2 [31] 12552-60 (2014).

12. H. An, D. Shin, S. M Choi, J. H. Lee, J. W. Son, B. K. Kim, H. J. Je, H. W. Lee, and K. J. Yoon, " $\mathrm{BaCeO}_{3}-\mathrm{BaZrO}_{3}$ Solid Solution (BCZY) as a High Performance Electrolyte of Protonic Ceramic Fuel Cells (PCFCs)," J. Korean Ceram. Soc., 51 [4] 271-77 (2014).

13. Y. Yoo and N. Lim, "Performance and Stability of Proton Conducting Solid Oxide Fuel Cells based on Yttriumdoped Barium Cerate-Zirconate Thin-Film Electrolyte," J. Power Sources, 229 48-57 (2013).

14. D. Buttrey, J. Sullivan, and A. Rheingold, "Phase Equilibrium Study of the $\mathrm{Y}-\mathrm{Ba}-\mathrm{NiO}_{5}$ System and Structural Characterization of the New Quasi-One-Dimensional Oxide $\mathrm{Y}_{2} \mathrm{BaNiO}_{5}$, J. Solid State Chem., 88 291-302 (1990).

15. Y. M. Guo, R. Ran, and Z. P. Shao, "Optimizing the Modification Method of Zinc-Enhanced Sintering of $\mathrm{BaZr}_{(0.4)} \mathrm{Ce}_{(0.4)} \mathrm{Y}_{(0.2)} \mathrm{O}_{(3-8)}$-based Electrolytes for Application in an Anode-Supported Protonic Solid Oxide Fuel Cell," Int. J. Hydrogen Energy, 35 [11] 5611-20 (2010).

16. S. Ricote and N. Bonanos, "Enhanced Sintering and Conductivity Study of Cobalt or Nickel Doped Solid Solution of Barium Cerate and Zirconate," Solid State Ionics, 181 [15-16] 694-700 (2010).

17. N. Nasani, D. Ramasamy, I. Antunes, B. Singh, and D. P. Fagg, "Structural and Electrical Properties of Strontium Substituted $\mathrm{Y}_{2} \mathrm{BaNiO}_{5}$," J Alloys Compd., 620 91-6 (2015). 